\title{
TEM Observations on the Role of Defects in Melting, Migration and Transformation of $\mathbf{P b}$-rich Precipitates in Al
}

\author{
U. Dahmen*, T. Radetic*, S. Hagège**, L. Zhang* and E. Johnson*** \\ *National Center for Electron Microscopy, LBNL, University of California, Berkeley, CA \\ **CNRS-CECM, Vitry-sur-Seine, France \\ ***Niels Bohr Institute, University of Copenhagen, Denmark
}

Defects play a key role in the thermodynamics and kinetics of precipitates in a solid matrix. However, there is little direct evidence on the specific role of defects in the evolution of individual precipitates. In this work, we have used in-situ transmission electron microscopy to study the thermal evolution of nanoscale $\mathrm{Pb}$ precipitates in $\mathrm{Al}$ during heating or cooling around the bulk melting point of $\mathrm{Pb}$. Quantitative analysis of these observations allows us to determine rates and mechanisms of melting, strain accommodation, migration and shape transformation of small $\mathrm{Pb}$ particles embedded in solid Al. The results shed new light on the role of defects in the behavior of small particles in solids.

$\mathrm{Al}-\mathrm{Pb}$ alloys form a simple monotectic system with a miscibility gap in the liquid state and negligible solubility in the solid state. Both components are fcc in structure and the preferred orientation of solid $\mathrm{Pb}$ inclusions in $\mathrm{Al}$ is a parallel-cube alignment of the two crystal lattices. For this orientation relationship, the interface energy is strongly anisotropic, resulting in a sharply faceted cuboctahedral equilibrium shape made of eight $\{111\}$ and six $\{100\}$ facets.

Figure 1 shows a sequence of micrographs taken from a video recording of a $\mathrm{Pb}-\mathrm{Cd}$ inclusion during cooling from the liquid state. All images were taken under 2-beam bright field conditions with the $\{111\}$ planes in diffracting condition. In (a) the precipitate is seen in the liquid state just before solidification. Immediately after solidification (b), large strain lobes indicate an elastic distortion consistent with a $\sim 3 \%$ volume contraction upon solidification [1]. Over the next 20 seconds the strain field is seen to decay steadily (c) until the particle again appears free of strain (d). The rate of decay varies strongly with temperature. Current efforts are underway to analyze this behavior in terms of the generation and diffusion of lattice vacancies.

Figure 2 illustrates the 1-dimensional motion of small liquid $\mathrm{Pb}$ inclusions trapped on a dislocation in Al. At the temperature of this experiment, the large liquid particle is immobile while the small liquid particles undergo rapid thermal motion. This motion has been analyzed quantitatively by tracing the trajectories of individual particles over extended time periods and performing statistical tests for the type and rate of motion. These observations can be used to determine particle diffusion coefficients, the interaction between particles and the mechanism of trapping on the dislocation [2].

Figure 3 shows a $\mathrm{Pb}$ inclusion on a grain boundary in Al during gradual heating toward and past the bulk melting point of $\mathrm{Pb}\left(327^{\circ} \mathrm{C}\right)$. As seen from the disappearance of the moiré fringes in a thin layer, at $322^{\circ} \mathrm{C}$, a premelted liquid layer has formed along the interface with the upper grain (a). The thickness of the melted layer increases with further approach to the melting point (b). The particle melts entirely, transforming to a lens shape (c), when the bulk melting point is traversed. A schematic of the premelting sequence in (d) shows that the premelted layer depends reversibly on temperature and curvature. These observations illustrate directly the effect of vacancies, dislocations and grain boundaries on the remarkably varied behavior of small $\mathrm{Pb}$ inclusions in $\mathrm{Al}[3]$.

\section{References}

[1] S. Hagège and U. Dahmen, Phil. Mag. Lett., 74 (1996) 259

[2] E. Johnson et al., Mater. Sci. and Eng. A, in press 
[3] This work was supported by the Director, Office of Basic Energy Sciences, Materials Science Division, US Department of Energy, under contract DE-AC3-76SF00098.
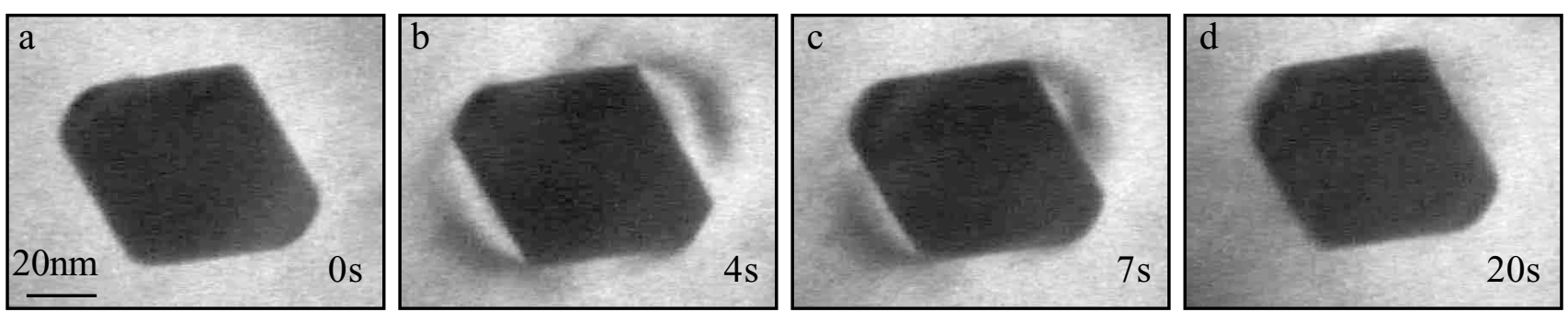

FIG.1 - In-situ observation of the solidification at about $219^{\circ} \mathrm{C}$ of a $\mathrm{Pb}-\mathrm{Cd}$ inclusion during cooling, establishment of an elastic strain field and its decay over the next 20 seconds.
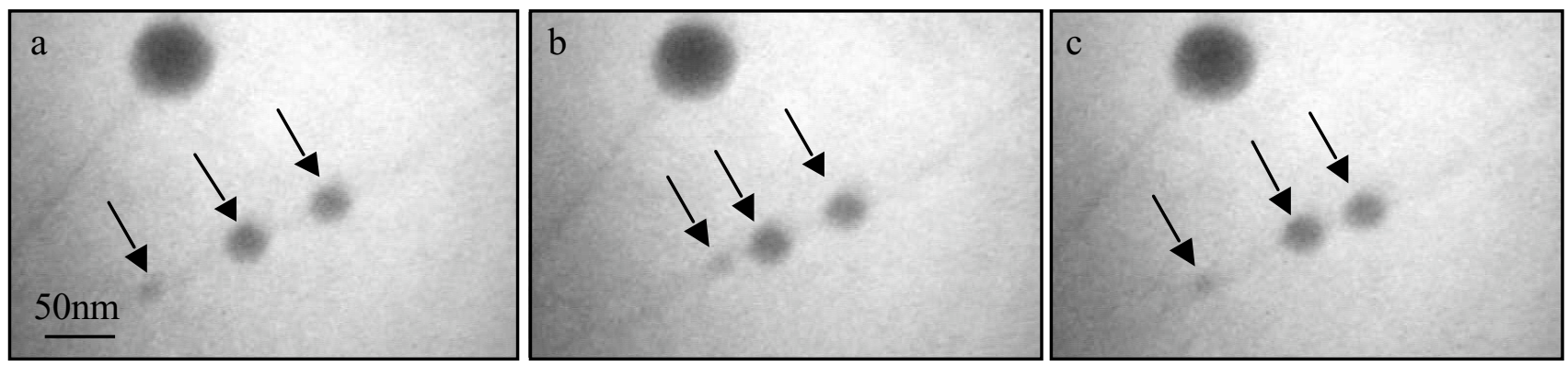

FIG.2 - In-situ observation of 1-dimensional Brownian motion of three liquid $\mathrm{Pb}$ inclusions (see arrows) attached to a dislocation in Al (faintly visible as diagonal line). At the temperature of observation $\left(446^{\circ} \mathrm{C}\right)$, all four inclusions are liquid, but only the smallest particles are seen to move.
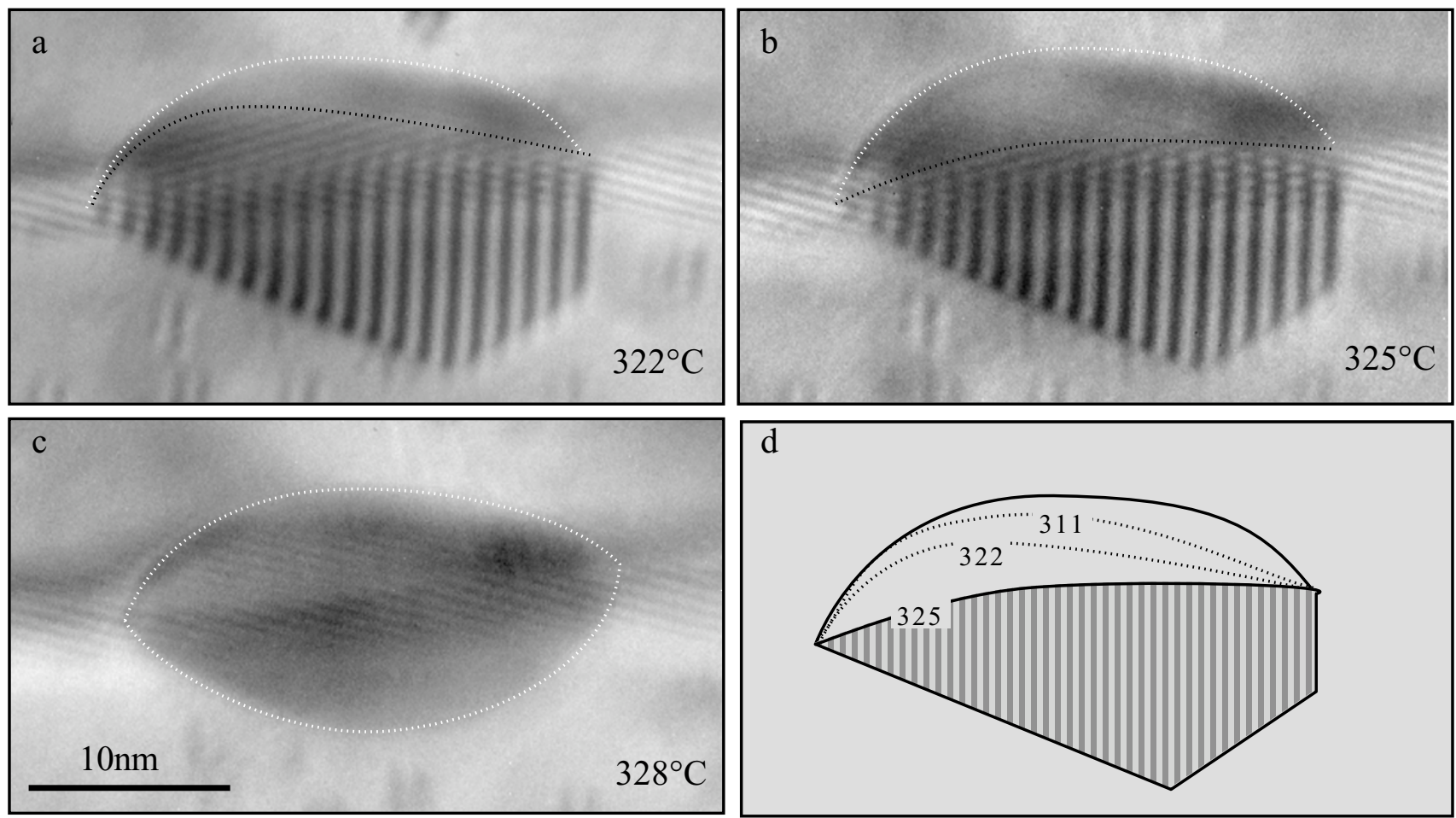

FIG.3 - Evidence of premelting during heating of a $\mathrm{Pb}$ precipitate on a grain boundary in Al. Melting (outlined) begins at the rounded interface with the upper grain. The schematic shows the reversible position of the melt front as a function of temperature. 